\title{
Detecting and modelling the magnetic field of the $\beta$ Cephei star V 2052 Ophiuchi ${ }^{\star}$
}

\author{
C. Neiner ${ }^{1}$, E. Alecian ${ }^{1}$, M. Briquet ${ }^{1,2, \star \star}$, M. Floquet $^{3}$, Y. Frémat ${ }^{4}$, C. Martayan ${ }^{5}$, \\ O. Thizy ${ }^{6}$, and the MiMeS collaboration
}

\author{
${ }^{1}$ LESIA, Observatoire de Paris, CNRS UMR 8109, UPMC, Université Paris Diderot, 5 place Jules Janssen, 92190 Meudon, France \\ e-mail: coralie.neiner@obspm.fr \\ 2 Instituut voor Sterrenkunde, K.U. Leuven, Celestijnenlaan 200D, 3001 Leuven, Belgium \\ 3 GEPI, Observatoire de Paris, CNRS UMR 8111, Université Paris Diderot, 5 place Jules Janssen, 92190 Meudon, France \\ 4 Royal Observatory of Belgium, 3 avenue circulaire, 1180 Brussels, Belgium \\ 5 ESO, Alonso de Cordova 3107, Vitacura, Casilla 19001, Santiago 19, Chile \\ ${ }^{6}$ Shelyak Instruments, Les Roussets, 38420 Revel, France
}

Received 24 August 2011 / Accepted 5 December 2011

\section{ABSTRACT}

\begin{abstract}
Aims. Following the indirect detection of a magnetic field in the $\beta$ Cephei star V 2052 Oph by Neiner and collaborators in 2003 with the Musicos spectropolarimeter, we remeasured the magnetic field of this star to attempt to directly confirm the detection of a magnetic field and study its configuration in greater detail.

Methods. We used the Narval spectropolarimeter installed at TBL (Pic du Midi, France), which is about 20 times more efficient than the Musicos spectropolarimeter. We applied the least-squares deconvolution (LSD) technique to various groups of lines to measure the circular polarisation of the light coming from V $2052 \mathrm{Oph}$. We synthesized the measured Stokes $V$ profiles with a centred and off-centred dipole model.

Results. For the first time, we clearly detect the Zeeman signature in the Stokes $V$ profiles of V 2052 Oph and thus directly prove the presence of a magnetic field in this star. The modulation with the rotation period is also confirmed and reflects an oblique dipole field. Thanks to the small error bars on the measurements, we are able to study the behaviour of different groups of lines and the centring of the dipole in the star. We find that the dipole is most likely off-centred along the magnetic axis and that He spots are present at the surface next to the magnetic axis.

Conclusions. We conclude that V $2052 \mathrm{Oph}$ is a magnetic He-strong $\beta$ Cep star, with a dipole field, probably off-centred, with $B_{\mathrm{pol}} \sim$ $400 \mathrm{G}$ and $\mathrm{He}$ patches close to the magnetic poles.
\end{abstract}

Key words. stars: magnetic field - stars: early-type - stars: individual: V 2052 Oph - starspots

\section{Introduction}

The $\beta$ Cephei star V 2052 Oph (HD 163472, HR 6684) is a bright $(V=5.85)$ B2 IV-V star with $v \sin i \sim 60 \mathrm{~km} \mathrm{~s}^{-1}$ and $P_{\text {rot }}=$ $3.638833 \pm 0.000003 \mathrm{~d}$, which is slightly enriched in $\mathrm{He}$ (Morel et al. 2006; Neiner et al. 2003a, hereafter N03). The presence of a magnetic field in this star has been suspected from the periodic variation of UV resonance lines observed with IUE (N03), which are similar to those observed in stars hosting an oblique magnetic dipole field. Measurements of this field with the Musicos spectropolarimeter at TBL (Pic du Midi, France) by N03 provided a possible indirect detection of the field: the signal-to-noise ratio was too weak to directly observe a Zeeman signature in the Stokes $V$ measurements but a rotational modulation of the stellar circular polarisation was detected in phase with the UV variations and rotation.

Since then, a new generation of spectropolarimeters, ESPaDOnS and Narval, has been installed on the CFHT 4-m and TBL 2-m telescopes. These spectropolarimeters are about 20 times more efficient than Musicos at TBL. To definitely

\footnotetext{
* Based on observations obtained with the Narval spectropolarimeter at the Télescope Bernard Lyot, Observatoire du Pic du Midi, France.

$\star \star$ Postdoctoral Fellow of the Fund for Scientific Research, Flanders.
}

establish the presence of a magnetic field in V $2052 \mathrm{Oph}$ and better study the configuration of the field, we therefore observed V 2052 Oph with Narval at TBL. The observations are presented in Sect. 2, the magnetic field is measured (Sect. 3) and modelled (Sect. 4), and the results are discussed in Sect. 5.

\section{Spectropolarimetric observations and methods}

The Narval spectropolarimeter has been installed at TBL (Pic du Midi, France) at the end of 2006. Data of V 2052 Oph have been collected from July 4 to 12, 2007, March 11 to April 13, 2009, and March 22 to August 12, 2010 (see Table 1). The rotation period of V 2052 Oph being 3.638833 days, these data cover all phases of rotation.

The polarimeter of Narval is attached at the Cassegrain focus of the TBL telescope while the echelle spectrograph is locked in a temperature-controlled box. The spectra collected with Narval span the wavelength domain from 3750 to $10500 \AA$ at a resolving power of about 65000 spread over 40 echelle orders. For the observations presented here, Narval was used with the normal CCD readout mode (40 s reading time), which is optimized for stars with $4 \leq V \leq 10$. This mode provides a gain of $1.4 \mathrm{e}^{-}$and a noise of $3.8 \mathrm{e}^{-} /$pixel. 
Table 1. Journal of Narval/TBL observations of V 2052 Oph obtained in 2007,2009 , and 2010 .

\begin{tabular}{|c|c|c|c|c|c|}
\hline \# & Date & $\begin{array}{c}\text { Mid-UT } \\
\text { h:min }\end{array}$ & $\begin{array}{c}\text { Mid-HJD } \\
-2450000\end{array}$ & $\begin{array}{c}T_{\exp } \\
\mathrm{s}\end{array}$ & Phase \\
\hline 1 & $2007-07-04$ & $21: 07$ & 4286.38 & $4 \times 420$ & 0.898 \\
\hline 2 & & $21: 38$ & 4286.41 & $4 \times 420$ & 0.904 \\
\hline 3 & 2007-07-05 & 02:01 & 4286.59 & $4 \times 420$ & 0.954 \\
\hline 4 & & $21: 13$ & 4287.39 & $4 \times 420$ & 0.174 \\
\hline 5 & & $21: 45$ & 4287.41 & $4 \times 420$ & 0.180 \\
\hline 6 & & $22: 16$ & 4287.43 & $4 \times 420$ & 0.186 \\
\hline 7 & & $22: 48$ & 4287.45 & $4 \times 420$ & 0.192 \\
\hline 8 & & $23: 25$ & 4287.48 & $4 \times 420$ & 0.199 \\
\hline 9 & & $23: 56$ & 4287.50 & $4 \times 420$ & 0.205 \\
\hline 10 & 2007-07-06 & $00: 27$ & 4287.52 & $4 \times 420$ & 0.211 \\
\hline 11 & & $00: 59$ & 4287.55 & $4 \times 420$ & 0.217 \\
\hline 12 & & 02:11 & 4287.60 & $4 \times 420$ & 0.231 \\
\hline 13 & & $02: 43$ & 4287.62 & $4 \times 420$ & 0.237 \\
\hline 14 & & $22: 05$ & 4288.42 & $4 \times 420$ & 0.459 \\
\hline 15 & & $22: 36$ & 4288.45 & $4 \times 420$ & 0.465 \\
\hline 16 & & $23: 10$ & 4288.47 & $4 \times 420$ & 0.471 \\
\hline 17 & & $23: 42$ & 4288.49 & $4 \times 420$ & 0.477 \\
\hline 18 & 2007-07-07 & $00: 14$ & 4288.51 & $4 \times 420$ & 0.483 \\
\hline 19 & & $00: 45$ & 4288.54 & $4 \times 420$ & 0.489 \\
\hline 20 & & $02: 26$ & 4288.61 & $4 \times 410$ & 0.508 \\
\hline 21 & & $02: 57$ & 4288.63 & $4 \times 410$ & 0.514 \\
\hline 22 & & $20: 54$ & 4289.38 & $4 \times 430$ & 0.720 \\
\hline 23 & & $21: 26$ & 4289.40 & $4 \times 430$ & 0.726 \\
\hline 24 & 2007-07-11 & $20: 25$ & 4293.36 & $4 \times 430$ & 0.814 \\
\hline 25 & & $20: 57$ & 4293.38 & $4 \times 430$ & 0.820 \\
\hline 26 & & $22: 27$ & 4293.44 & $4 \times 420$ & 0.837 \\
\hline 27 & & $22: 58$ & 4293.46 & $4 \times 420$ & 0.843 \\
\hline 28 & & $23: 30$ & 4293.48 & $4 \times 420$ & 0.849 \\
\hline 29 & 2007-07-12 & $02: 33$ & 4293.61 & $4 \times 420$ & 0.884 \\
\hline 30 & & $21: 22$ & 4294.39 & $4 \times 420$ & 0.099 \\
\hline 31 & & $23: 47$ & 4294.50 & $4 \times 440$ & 0.127 \\
\hline 32 & $2009-03-12$ & $04: 52$ & 4902.70 & $4 \times 300$ & 0.270 \\
\hline 33 & 2009-03-17 & $04: 25$ & 4907.68 & $4 \times 300$ & 0.639 \\
\hline 34 & 2009-04-14 & $01: 35$ & 4935.57 & $4 \times 420$ & 0.302 \\
\hline 35 & & $02: 10$ & 4935.59 & $4 \times 420$ & 0.309 \\
\hline 36 & & $02: 43$ & 4935.62 & $4 \times 420$ & 0.315 \\
\hline 37 & $2010-03-23$ & $04: 31$ & 5278.69 & $4 \times 420$ & 0.596 \\
\hline 38 & 2010-04-17 & 00:03 & 5303.50 & $4 \times 420$ & 0.416 \\
\hline 39 & 2010-07-01 & $23: 20$ & 5379.48 & $4 \times 420$ & 0.294 \\
\hline 40 & $2010-07-05$ & $21: 59$ & 5383.42 & $4 \times 420$ & 0.378 \\
\hline 41 & 2010-07-13 & $21: 03$ & 5391.38 & $4 \times 420$ & 0.566 \\
\hline 42 & 2010-07-19 & 03:03 & 5396.63 & $4 \times 420$ & 0.009 \\
\hline 43 & $2010-07-25$ & $23: 15$ & 5403.47 & $4 \times 420$ & 0.889 \\
\hline 44 & $2010-08-12$ & 21:07 & 5421.38 & $4 \times 420$ & 0.811 \\
\hline
\end{tabular}

Notes. Column 1 indicates the number of the polarimetric sequence. Columns 2 and 3 show the date and time of the middle of observations, while Col. 4 gives the Heliocentric Julian Date (HJD) at the middle of observations. Column 5 gives the exposure time in seconds for each sequence, and Col. 6 the rotational phase using $P_{\text {rot }}=3.638833 \mathrm{~d}$ and $\mathrm{HJD}_{0}=2447383.89$.

We measured the circular polarisation of the stellar light. Each of the polarimetric measurements consists of a sequence of four subexposures with the same exposure time, between 300 and $420 \mathrm{~s}$ each. A full polarimetric measurement therefore takes about half an hour (maximum $4 \times 420$ s plus $4 \times 40$ s for reading the CCD camera). The crosstalk between circular and linear polarisations in Narval has been measured during the commissioning of Narval and is lower than $1 \%$.

Usual bias, flat-fields, and ThAr calibrations were obtained at the beginning and at the end of each night. The data reduction was performed using LIBRE-ESPRIT, the dedicated reduction software available at TBL. LIBRE-ESPRIT is based on ESPRIT, the software developed by Donati et al. (1997) for the Musicos spectropolarimeter, and adapted to the instrumental configuration of Narval.

The least-squares deconvolution (LSD) technique (Donati et al. 1997) was used to average the spectral line profiles and the Stokes $V$ signature of each measurement. This allows one to obtain an increased signal-to-noise for the detection of a magnetic field. For the LSD, four line masks were prepared according to the Narval spectrum of V 2052 Oph: one mask containing 374 photospheric lines of various chemical elements, one mask containing the $50 \mathrm{He}$ lines present in the spectrum, one mask with all considered photospheric lines except the He lines, i.e. with 324 lines, and one mask with all $20 \mathrm{Si}$ lines. These numbers should be compared with the 159 lines used in the LSD mask for Musicos measurements (N03). Note that the LSD method assumes that the intrinsic broadening of each line is similar, therefore $\mathrm{H}$ lines are never used in LSD line masks. For each spectral line, the mask contains the wavelength, depth, and Landé factor, to be used by the LSD programme. Landé factors were extracted from Kurucz lists of spectral lines ${ }^{1}$. Line depths were adjusted to the observed spectrum.

For each spectrum and each mask, LSD Stokes $I$ and $V$ profiles were extracted together with the corresponding sum weights. These profiles and weights were used to calculate the longitudinal magnetic field and fit Stokes profiles, except for the mask with only Si lines for which the signal-to-noise ratio is too low because of the low amount of available lines (see Fig. 2).

\section{Magnetic field measurements}

\subsection{Observed Zeeman signatures}

For the 44 Narval measurements, Fig. 1 shows the LSD Stokes $I$ and $V$ profiles (top panel) as well as colourscales (bottom panel) of the residual LSD Stokes $I$ spectra (compared to the averaged LSD Stokes $I$ spectrum) and of the LSD Stokes $V$ spectra. For the first time, a Zeeman signature is clearly detected in the Stokes $V$ profiles of V 2052 Oph and the rotation modulation can clearly be observed in the colourscale of the LSD Stokes $V$ spectra (right panel of Fig. 1).

In contrast, the null diagnosis $N$, which is calculated with the LSD by combining the subexposures of a polarimetric sequence in a different way than for Stokes $V$ and which gives an indication of the pollution of the measurement by non-stellar effects, shows no signature (see Fig. 2) and gives values between 0 and $\sim 30 \mathrm{G}$ with error bars similar to those obtained for the longitudinal field (see Table 2). This confirms that the observed $\mathrm{V}$ polarisation signature indeed originates from the star. We thus confirm the presence of a magnetic field in V 2052 Oph.

In the LSD I profiles, variations are observed as well (see left panel of Fig. 1) but those variations are not in phase with the rotation. For example, the $I$ profiles at rotational phases 0.126 and 0.514 are very similar, but the successive profiles around phase 0.89 are very different. The variation observed here corresponds to the pulsations of the star with $P=0.14$ and $0.15 \mathrm{~d}$ for the main modes (see N03 and Briquet et al., in prep.).

If we look at the Zeeman signatures using specific chemical species instead of all lines in the LSD, we note that the signature can differ from one type of lines to the others. For example He lines and Si lines have a different behaviour (see examples in Fig. 2). The LSD profiles calculated with all photospheric lines

\footnotetext{
${ }^{1}$ http://kurucz.harvard.edu
} 

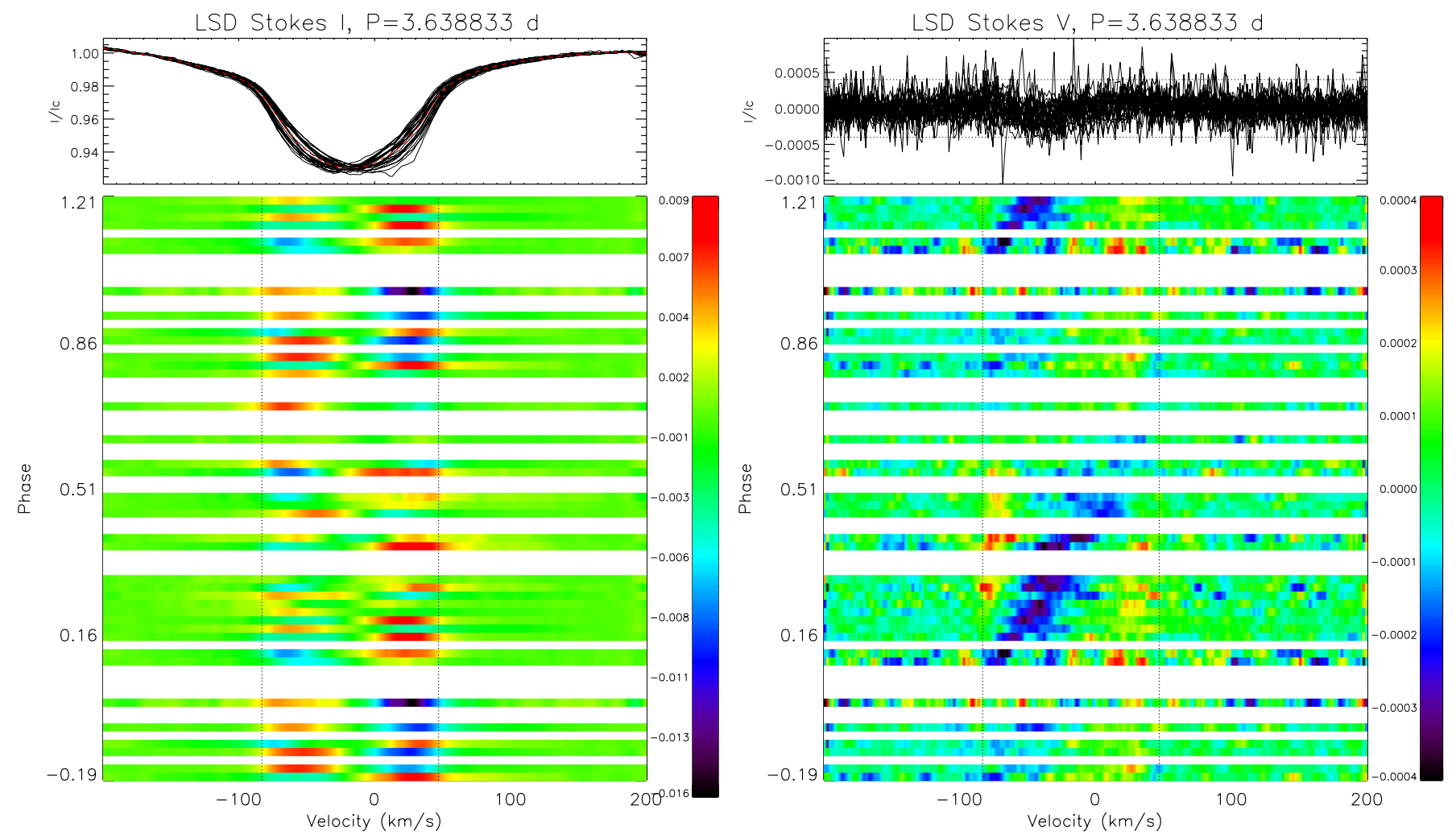

Fig. 1. LSD I profiles (left) and LSD Stokes $V$ profiles (right) of V 2052 Oph observed with Narval. Top: the profiles themselves. The averaged $I$ profile is also shown (dashed red) in the left panel. Bottom: a colourscale of the variation with phase. The phase is folded with $\mathrm{HJD}_{0}=2447383.89$ and $P_{\text {rot }}=3.638833$ d. For the LSD $I$ profiles, the colourscale shows residual spectra compared to the averaged profile. The vertical dashed lines indicate $\pm v \sin i$. The zero velocity point corresponds to the laboratory wavelength reference while the profiles are centred on the stellar radial velocity value.

present in the spectrum (except hydrogen, see above) are similar to the profiles calculated with the He lines only. The LSD profiles calculated with all lines but He differ from those. See Figs. 5 to 7.

\subsection{Measured longitudinal magnetic field}

The measurement of the longitudinal magnetic field from an LSD Stokes $V$ profile requires one to set the range over which the magnetic field should be integrated. Using a range narrower than the width of the line will corrupt the magnetic field values, while using a range that is too wide will artificially increase their error bars.

To determine the integration range we first calculated the magnetic field values using a quite wide range $v= \pm 90 \mathrm{~km} \mathrm{~s}^{-1}$ around the line centre. This first estimate allows us to pinpoint which measurements were taken close to maximum or minimum of the longitudinal magnetic field, i.e. close to a phase where one of the magnetic poles is seen best. We singled out measurements 6 and 12 as probable observations close to minimum field. We then calculated the field for these two measurements using different integration ranges. If these measurements were indeed taken at minimum field, the value of the field decreases as we increase the size of the integration range from a low value to its correct width. If we increase the size of the integration range above the correct width, the value of the field does not change anymore but the error bars increase. Figure 3 shows the values and error bars obtained for the two measurements 6 and 12. This figure suggests that the adequate integration range is about $\pm 65 \mathrm{~km} \mathrm{~s}^{-1}$. This range is reported in Fig. 2 and corresponds well to the known $v \sin i \sim 60 \mathrm{~km} \mathrm{~s}^{-1}$ (N03). Note, however, that using a range between 55 and $90 \mathrm{~km} \mathrm{~s}^{-1}$ does not change the conclusions presented here.

Table 2 provides the phases of observation with $P_{\text {rot }}=$ $3.638833 \mathrm{~d}$ and $\mathrm{HJD}_{0}=2447383.89$, the longitudinal magnetic field values $B$ obtained using an integration range of $\pm 65 \mathrm{~km} \mathrm{~s}^{-1}$, and the error bars $\sigma B$ on these values. Values are given applying LSD on all photospheric lines, on He lines only, or on all lines except He lines. The mask with $\mathrm{Si}$ lines only is not considered here because of the low signal-to-noise ratio in the LSD profiles. The error bars are typically $20-25 \mathrm{G}$ for all lines or $\mathrm{He}$ lines only, and $45 \mathrm{G}$ when rejecting He lines. This should be compared with the $\sim 200 \mathrm{G}$ error bars obtained on Musicos measurements using all lines (N03). Narval thus allows one to reach error bars that are 10 times better than Musicos. For the phases we use $\mathrm{HJD}_{0}=2447383.89$ as a reference because this is the time of minimum equivalent width of the wind-sensitive UV line and the ephemeris reference for V 2052 Oph (see N03).

\subsection{Search for periodicity}

From the IUE observations of wind-sensitive resonance lines of V $2052 \mathrm{Oph}$, we know that the period of rotation of this star is $P_{\text {rot }}=3.638833 \mathrm{~d}\left(\mathrm{~N} 03 ; f_{\text {rot }}=0.2748 \mathrm{c} \mathrm{d}^{-1}\right)$. We nevertheless search for the modulation period of the longitudinal magnetic field of V $2052 \mathrm{Oph}$. This search for periodicity is not easy with only 44 measurements spread over three years and the results should therefore be interpreted with care. It is possible, however, to compare them with the known value of the rotation period. 
Rotation phase

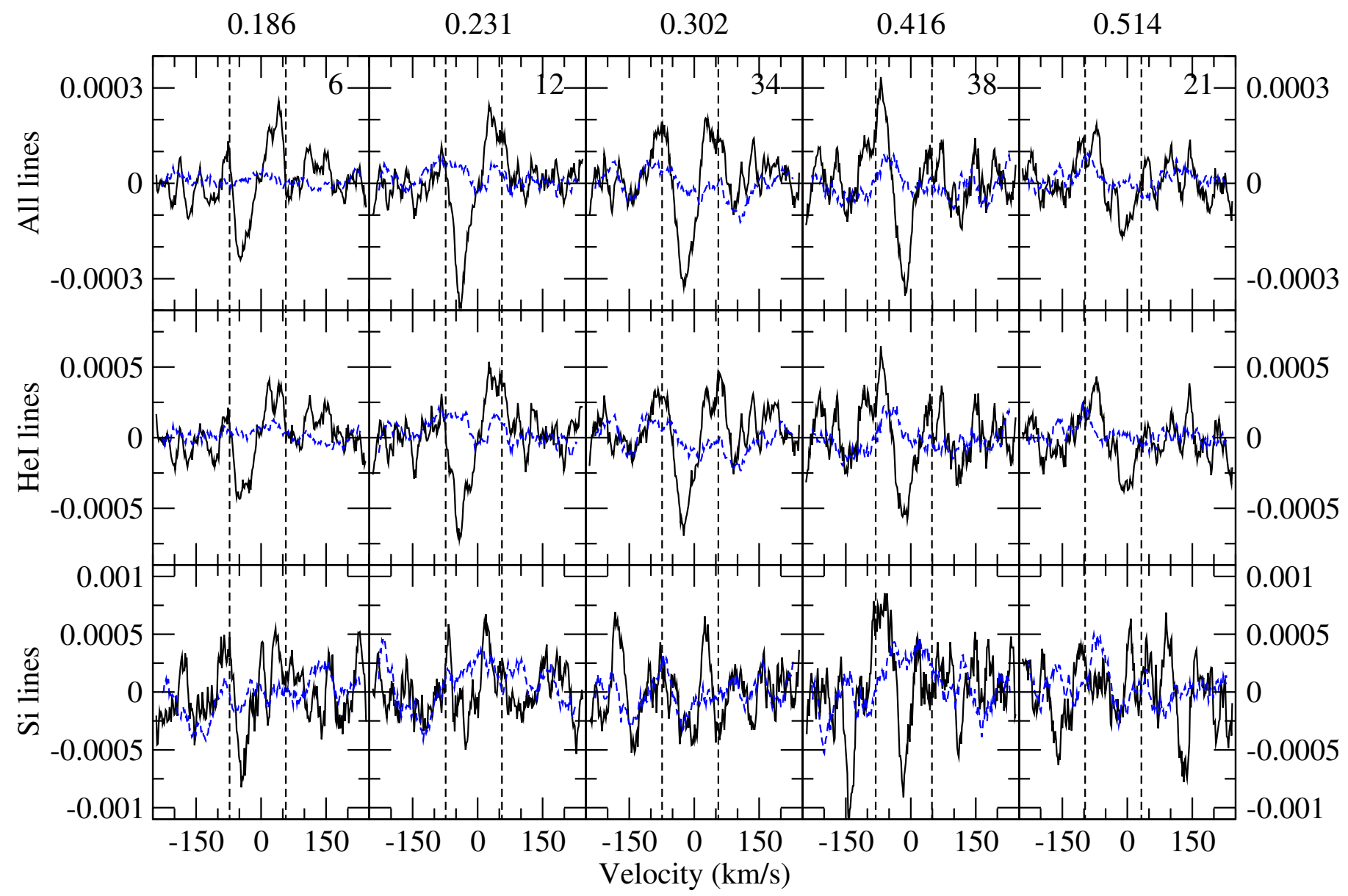

Fig. 2. LSD Stokes $V$ (solid black line) and null diagnosis $N$ (dashed blue line) profiles calculated for groups of lines from different chemical species (top to bottom) and at typical rotation phases (left to right). The number of the polarimetric sequence is indicated in the top panel. Vertical dashed lines show the integration range at $\pm 65 \mathrm{~km} \mathrm{~s}^{-1}$ centred on the minimum of the Stokes $I$ profiles.

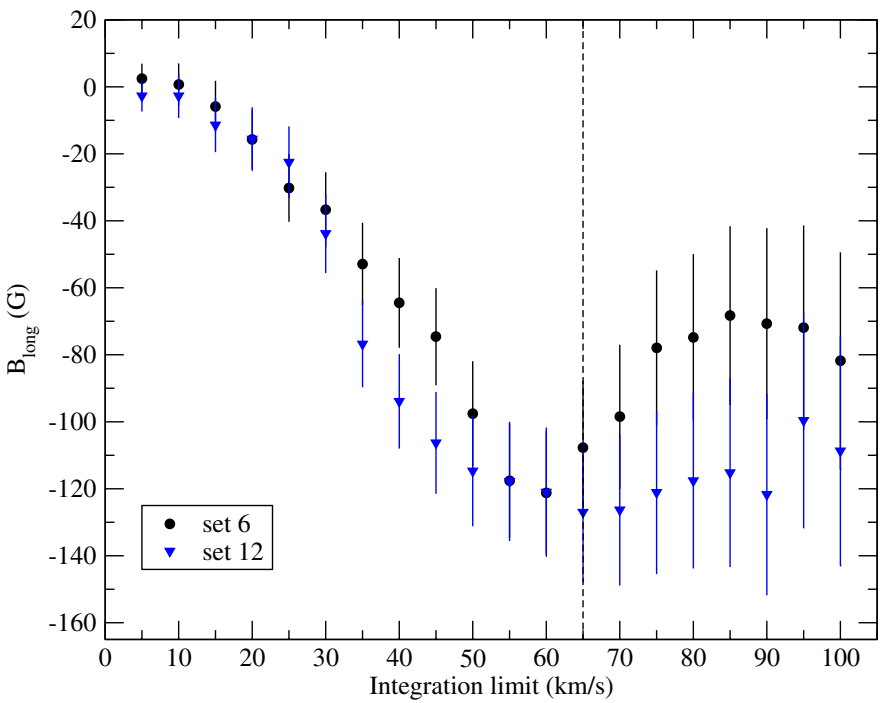

Fig. 3. Longitudinal magnetic field values obtained for measurements 6 and 12 for different half-widths of the integration range for the LSD Stokes $V$ signature.

Since the window function is far from optimal for a periodicity search, we used a clean algorithm, that can account for the sparsity of the datapoints. We obtained $f=0.286 \mathrm{c} \mathrm{d}^{-1}$ $(P=3.5 \mathrm{~d})$, which is close to the known rotation frequency $f_{\text {rot }}$ indicated above. We also obtained a second frequency $f=$ $7.08 \mathrm{c} \mathrm{d}^{-1}(P=0.14 \mathrm{~d})$, which is close to the main known radial pulsation frequencies of V $2052 \mathrm{Oph}: f_{\text {puls }}=7.15 \mathrm{c} \mathrm{d}^{-1}$ (N03; Briquet et al., in prep.).

The Stokes $V$ measurements are not affected by the changes of the I profiles caused by pulsations during a polarimetric sequence thanks to the short exposure time $(\sim 30$ min per polarimetric sequence to be compared with the $\sim 200 \mathrm{~min}$ of the pulsation period) and as witnessed by the null diagnostic $N$ flat profiles (Fig. 2). However, two polarimetric measurements taken at the same rotation phase but different pulsation phases have different I profiles and consequently slightly different $\mathrm{V}$ profiles. This is probably why the pulsation period is observed in the magnetic data. Nevertheless, only the shape of the profiles changes, not the determination of the longitudinal magnetic field values.

We used the known and very precise value of the rotation period $P_{\text {rot }}=3.638833 \mathrm{~d}$ determined from the UV variations (N03). This period is confirmed from a spectroscopic pulsation and seismology study by Briquet et al. (in prep.).

\section{Magnetic configuration}

\subsection{Modelling the longitudinal field}

We plot in Fig. 4 the longitudinal magnetic field values and corresponding error bars obtained above for each group of lines 
Table 2. Magnetic field measurements of V $2052 \mathrm{Oph}$ obtained with Narval in 2007, 2009, and 2010.

\begin{tabular}{|c|c|c|c|c|c|c|c|}
\hline \multirow{3}{*}{ \# } & \multirow{3}{*}{ Phase } & \multicolumn{2}{|l|}{ All lines } & \multicolumn{2}{|l|}{$\overline{\text { He lines }}$} & \multicolumn{2}{|l|}{ All but $\mathrm{He}$} \\
\hline & & B & $\sigma B$ & $B$ & $\sigma B$ & $B$ & $\sigma B$ \\
\hline & & G & G & G & G & G & \\
\hline 1 & 0.89792 & -60.0 & 19.3 & -44.6 & 21.4 & -122.1 & $\overline{42.4}$ \\
\hline 2 & 0.90392 & -53.9 & 18.9 & -36.7 & 21.1 & -113.4 & 42.4 \\
\hline 3 & 0.95401 & -86.7 & 18.1 & -77.7 & 20.1 & -155.2 & 41.5 \\
\hline 4 & 0.17394 & -78.7 & 20.9 & -93.6 & 23.5 & 10.2 & 46.0 \\
\hline 5 & 0.17994 & -90.0 & 20.1 & -72.5 & 22.7 & -121.0 & 43.6 \\
\hline 6 & 0.18593 & -107.7 & 19.9 & -90.4 & 22.4 & -178.7 & 42.2 \\
\hline 7 & 0.19193 & -92.4 & 19.8 & -84.2 & 22.4 & -150.3 & 41.3 \\
\hline 8 & 0.19896 & -72.5 & 19.4 & -75.1 & 21.8 & -73.2 & 41.3 \\
\hline 9 & 0.20496 & -69.2 & 19.0 & -72.9 & 21.4 & -63.5 & 40.7 \\
\hline 10 & 0.21095 & -56.8 & 20.2 & -54.8 & 22.7 & -16.9 & 43.8 \\
\hline 11 & 0.21695 & -84.0 & 20.8 & -92.9 & 23.5 & -32.3 & 44.4 \\
\hline 12 & 0.23074 & -127.0 & 20.9 & -128.7 & 23.8 & -88.3 & 43.0 \\
\hline 13 & 0.23674 & -68.0 & 22.2 & -68.8 & 25.2 & -67.7 & 47.3 \\
\hline 14 & 0.45853 & 29.8 & 23.7 & 42.2 & 26.7 & 11.3 & 50.7 \\
\hline 15 & 0.46452 & -3.5 & 22.6 & 17.4 & 25.4 & -105.7 & 48.8 \\
\hline 16 & 0.47 & 41.5 & 19.7 & 15.5 & 22.5 & 132.4 & 42.1 \\
\hline 17 & 0.477 & 62.6 & 18.9 & 54.9 & 21.2 & 97.2 & 41.1 \\
\hline 18 & 0.48313 & 61.8 & 20.8 & 65.2 & 23.4 & 93.0 & 46.3 \\
\hline 19 & 0.48913 & 46.5 & 22.5 & 52.4 & 25.3 & 86.7 & 49.2 \\
\hline 20 & 0.50842 & 41.3 & 23.5 & 40.3 & 26.5 & 121.5 & 49.6 \\
\hline 21 & 0.51429 & 60.9 & 23.1 & 68.8 & 25.9 & 47.1 & 55.1 \\
\hline 22 & 0.71977 & -14.7 & 21.3 & -9.8 & 24.1 & -51.0 & 45.4 \\
\hline 23 & 0.72589 & -11.5 & 23.6 & 9.5 & 26.7 & -107.4 & 49.4 \\
\hline 24 & 0.81358 & -44.8 & 22.3 & -42.9 & 25.2 & -52.7 & 46.7 \\
\hline 25 & 0.81971 & -88.0 & 22.0 & -73.3 & 24.9 & -155.1 & 47.0 \\
\hline 26 & 0.8368 & -38.1 & 22.0 & -36.5 & 24.8 & -8.7 & 49.1 \\
\hline 27 & & -57.0 & 21.9 & -73.0 & 24.5 & -4.6 & 47.7 \\
\hline 28 & 0.848 & -75.6 & 19.9 & -53.6 & 22.6 & -198.8 & 42.0 \\
\hline 29 & & -50.3 & 18.5 & -64.6 & 20.5 & -6.8 & 40.8 \\
\hline 30 & & -88.6 & 39.7 & -81.2 & 42.3 & -44.2 & 97.2 \\
\hline 31 & 0.12690 & -86.0 & 32.3 & -87.2 & 35.9 & -94.3 & 74.3 \\
\hline$\overline{32}$ & 0 & -86.4 & 22.9 & -81.4 & 26.2 & -91.3 & 46. \\
\hline 33 & 0.63919 & 43.1 & 20.4 & 40.6 & 23.0 & 60.4 & 43.3 \\
\hline 34 & 0.3021 & -44.0 & 21.8 & -44.5 & 24.9 & -56.7 & 43.8 \\
\hline 35 & 0.3088 & 7.6 & 18. & 17.6 & 21.1 & -55.7 & 39.5 \\
\hline 36 & .31528 & -14.2 & 19.6 & -13.6 & 22.2 & -19.0 & 42.2 \\
\hline$\overline{37}$ & 0.59635 & 42.2 & 19.8 & 54.8 & 22.5 & -0.6 & 43.0 \\
\hline 38 & .41614 & 63.9 & 30.6 & 44.2 & 35.4 & 143.2 & 64.6 \\
\hline 39 & 0.29446 & -34.5 & 22.8 & -123.6 & 28.4 & 81.0 & 50.7 \\
\hline 40 & 37819 & 12.5 & 19.3 & -12.6 & 21.8 & 13.9 & 40.5 \\
\hline 41 & 0.56592 & 66.7 & 23.2 & 80.5 & 26.0 & -44.0 & 53.8 \\
\hline 42 & 0.00868 & -23.0 & 41.1 & 5.3 & 47.0 & -178.1 & 109.2 \\
\hline 43 & 0.88878 & -41.8 & 20.9 & -36.1 & 23.3 & -63.6 & 46.5 \\
\hline 44 & 0.81072 & -1.9 & 20.5 & 13.9 & 22.7 & -45.4 & 43.8 \\
\hline
\end{tabular}

Notes. Column 1 indicates the number of the polarimetric set. Column 2 gives the rotational phase using $P_{\text {rot }}=3.638833 \mathrm{~d}$ and $\mathrm{HJD}_{0}=$ 2447383.89 . The following columns provide the longitudinal magnetic field value $B$ and its error bar $\sigma B$ in Gauss, for LSD using all photospheric lines (Cols. 3 and 4), using He lines only (Cols. 5 and 6), and using all photospheric lines but He lines (Cols. 7 and 8).

versus the phases computed with the known rotation period and using $\mathrm{HJD}_{0}=2447383.89$. We then performed a dipole fit to the data, i.e. a sine fit of the form

$B(x)=B_{0}+B_{l} \times \sin \left(2 \pi\left(x+\phi_{\mathrm{d}}\right)\right)$.

The results we obtained for each group of lines are plotted in Fig. 4 and summarized in Table 3 .

The values of the reduced $\chi^{2}$ indicate that a dipole is a good fit to the data. The reduced $\chi^{2}$ value is less good when we consider all but He lines. This is directly related to the larger scatter

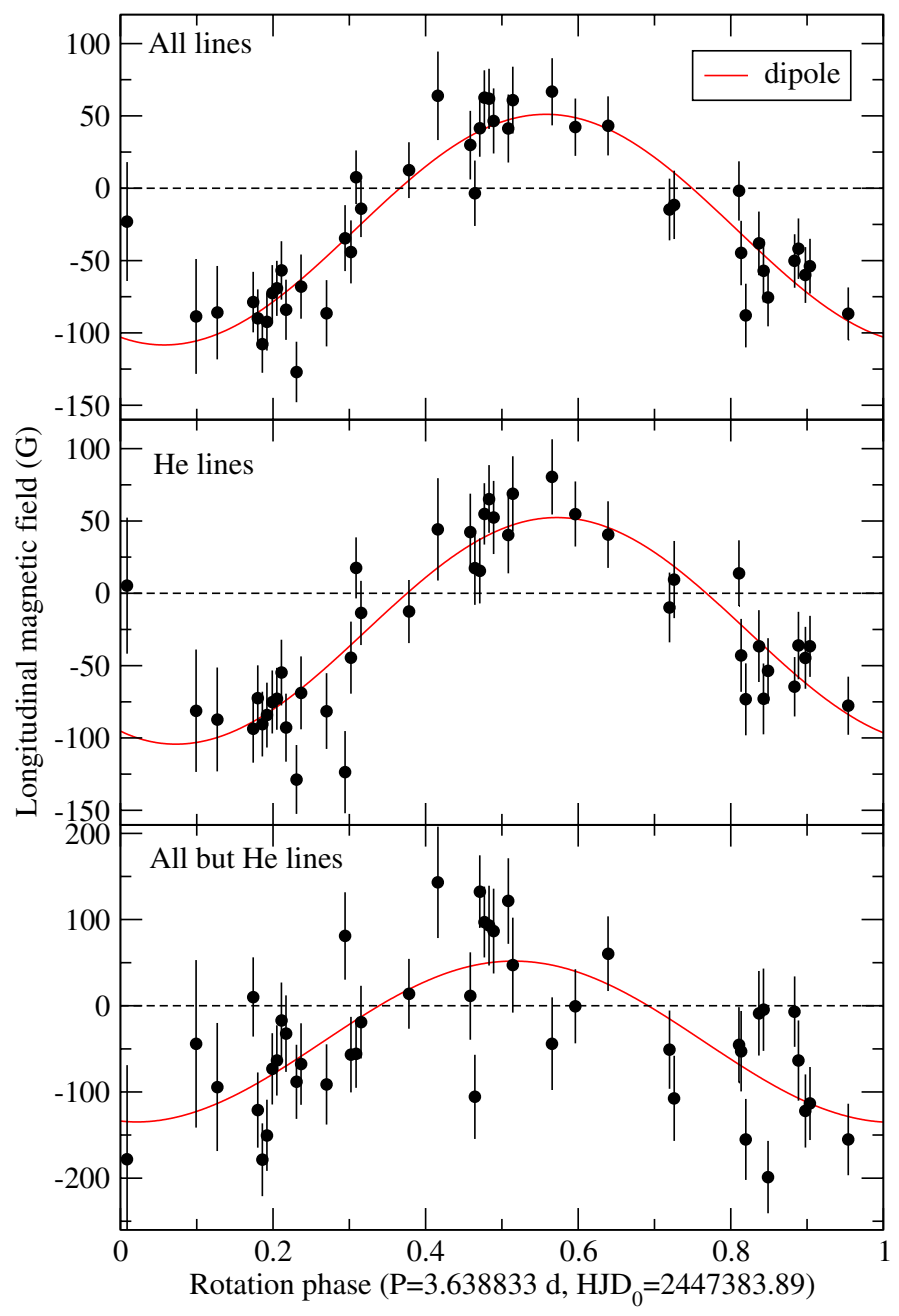

Fig. 4. Longitudinal magnetic field values plotted in phase with $P_{\mathrm{rot}}=$ $3.638833 \mathrm{~d}$ and $\mathrm{HJD}_{0}=2447383.89$ for the groups of lines: all photospheric lines (top panel), He lines only (middle panel), and all photospheric lines except He lines (bottom panel). A dipole fit of the data is superimposed in each panel.

observed in the bottom panel of Fig. 4. The value of $\phi_{\mathrm{d}}$, however, is shifted by 0.02 to 0.07 compared to the expected rotation phase $\left(\phi_{\mathrm{d}}=-0.25\right)$. The error bars on the ephemeris only allow for a 0.02 phase shift between the epochs when UV data and Narval data were obtained. Therefore the dipole fit using all but $\mathrm{He}$ lines is compatible with the ephemeris determined from the UV data, while the fit is not compatible when we used or included He lines. This suggests that He patches are present at the surface of the star and are slightly shifted compared to the magnetic axis. This is often observed in magnetic stars and is an indirect signature of the presence of magnetic field.

\subsection{Geometry of the field}

From their UV curve, N03 found that the obliquity angle of the dipole is $\beta=35 \pm 17^{\circ}$.

From the dipole fit of the Narval data presented here, using the inclination angle $i=71 \pm 10^{\circ}(\mathrm{N} 03)$, the equation

$r=\frac{B_{\min }}{B_{\max }}=\frac{\cos (i-\beta)}{\cos (i+\beta)}$

and measuring $r$ from the dipole fit for the 3 groups of lines, we found the obliquity angle $\beta$. Results are reported in Table 3. 
Table 3. Results of a dipole fit (see Eq. (1)) on the longitudinal magnetic field values using the three groups of lines.

\begin{tabular}{lccccccc}
\hline \hline & $B_{0}$ & $B_{1}$ & $\phi_{\mathrm{d}}$ & $\chi^{2}$ & $r$ & $\beta$ & $B_{\text {pol }}$ \\
& $\mathrm{G}$ & $\mathrm{G}$ & & & & $\mathrm{deg}$ & $\mathrm{G}$ \\
\hline All lines & $-29 \pm 3$ & $80 \pm 5$ & $-0.31 \pm 0.01$ & 1.19 & $-2.12_{-1.68}^{-2.73}$ & $44_{19}^{65}$ & $-408_{-175}^{1147}$ \\
He lines & $-26 \pm 4$ & $78 \pm 6$ & $-0.32 \pm 0.01$ & 1.25 & $-1.99_{-1.53}^{-2.65}$ & $46_{19}^{69}$ & $-387_{-163}^{1206}$ \\
All but He & $-42 \pm 7$ & $93 \pm 11$ & $-0.27 \pm 0.02$ & 1.87 & $-2.61_{-1.67}^{-4.57}$ & $38_{14}^{65}$ & $-529_{-134}^{001}$ \\
\hline
\end{tabular}

Notes. The first three columns indicate the parameters of the sine fit curve and the reduced $\chi^{2}$ value of the fit. The last three columns indicate the ratio $r$ (see Eq. (2)), corresponding obliquity angle $\beta$, and derived strength of the dipolar magnetic field $B_{\text {pol. }} 3 \sigma$ errors are reported for each parameter.

Table 4. Magnetic field configuration obtained by modelling LSD Stokes profiles with a centred or off-centred oblique dipole magnetic field.

\begin{tabular}{lccccccccc}
\hline \hline Lines & $\begin{array}{c}i \\
\mathrm{deg}\end{array}$ & $\begin{array}{c}\beta \\
\mathrm{deg}\end{array}$ & $\begin{array}{c}B_{\mathrm{pol}} \\
\mathrm{G}\end{array}$ & $\begin{array}{c}d \\
R\end{array}$ & $\Delta \psi$ & $\chi_{\min }^{2}$ & $\chi_{3 \sigma}^{2}$ & $\begin{array}{c}B^{+} \\
\mathrm{G}\end{array}$ & $\begin{array}{c}B^{-} \\
\mathrm{G}\end{array}$ \\
\hline All & $75 \pm 4$ & $27 \pm 3$ & $-338 \pm 33$ & - & $0.086 \pm 0.018$ & 0.5815 & 0.5848 & - & - \\
All & $67 \pm 5$ & $43 \pm 5$ & $-289 \pm 28$ & $0.19 \pm 0.05$ & $0.054 \pm 0.015$ & 0.5560 & 0.5598 & $171_{134}^{217}$ & $-543_{-402}^{-733}$ \\
$\mathrm{He}$ & $77 \pm 5$ & $25 \pm 4$ & $-261 \pm 37$ & - & $0.099 \pm 0.026$ & 0.4852 & 0.4886 & - & - \\
$\mathrm{He}$ & $69 \pm 5$ & $38 \pm 5$ & $-244 \pm 32$ & $0.26 \pm 0.07$ & $0.051 \pm 0.018$ & 0.4623 & 0.4660 & $122_{90}^{163}$ & $-602_{-399}^{-917}$ \\
All but He & $73 \pm 4$ & $35 \pm 4$ & $-560 \pm 60$ & - & $0.084 \pm 0.021$ & 0.8540 & 0.8582 & - & - \\
All but He & $53 \pm 8$ & $52 \pm 6$ & $-418 \pm 46$ & $0.18 \pm 0.04$ & $0.038 \pm 0.017$ & 0.8072 & 0.8118 & $254_{205}^{313}$ & $-758_{-585}^{-978}$ \\
\hline
\end{tabular}

Notes. Column 1 indicates which observed LSD profiles have been fitted, while the following seven columns show results from the models: the inclination angle $i$ (Col. 2), the obliquity angle $\beta$ (Col. 3), the polar field strength $B_{\text {pol }}$, the off-centring of the field along the magnetic axis $d$, the phase shift between the model and the observations $\Delta \psi$, the minimum reduced $\chi^{2}$ value of the multi-dimension surface, the reduced $\chi^{2}$ value with $3 \sigma$ errors on the parameters ( $99.7 \%$ reliability). $3 \sigma$ error bars are also given on the fitted parameters. The last two columns indicate the strength of the magnetic field at the positive and negative poles for the off-centred case.

The values of the obliquity angle are fully compatible with the value obtained from the UV wind lines. However, we found that the $\beta$ angle determined for He lines or other than He lines are slightly different (although compatible within the error bars). Again this points towards the presence of patches of He that are slightly shifted from the magnetic axes.

In addition, the value of $B_{0}$ and $B_{l}$ obtained with the Narval observations are much more precise than those obtained from Musicos (N03), and allow one to determine a new value of the polar field using the formula

$B_{0} \pm B_{l}=0.296 * B_{\mathrm{pol}} \cos (\beta \pm i)$

(when the limb-darkening coefficient is 0.4 , see Borra \& Landstreet 1980). The $B_{\text {pol }}$ values we obtained for the three groups of lines are shown in Table 3.

\subsection{Modelling the LSD Stokes profiles}

The longitudinal field values provide information that is integrated over the visible hemisphere of the star. To obtain more details on the magnetic configuration, it is necessary to model Stokes $V$ profiles.

We have shown above that a dipole field is a good representation of the observed magnetic field. We therefore used an oblique rotator model to calculate Stokes $V$ profiles. We first modelled a centred dipole, then a dipole off-centred along the magnetic axis by an amount $d$.

We did not perform radiative magnetised transfer here. We used Gaussian local intensity profiles with a width calculated according to the resolving power of Narval and a macroturbulence value of $20 \mathrm{~km} \mathrm{~s}^{-1}$ as determined by Morel et al. (2006). The depth of the intensity profile was determined by fitting the observed LSD I profiles. We then calculated local Stokes $V$ profiles assuming the weak-field case and integrated over the visible hemisphere of the star. We obtained synthetic LSD Stokes $V$ profiles, which we normalized to the intensity continuum.

We used the weighted mean Landé factor and wavelength derived from the LSD mask applied to the Narval observations and the rotation period and ephemeris determined from UV data by N03. The fit includes four or five parameters: $i, \beta, B_{\mathrm{pol}}$, a phase shift compared to the UV ephemeris $\Delta \psi$, and the off-centring distance $d$ in the off-centred case ( $d=0$ for a centred dipole and $d=1$ if the centre of the dipole is at the surface of the star).

To increase the signal-to-noise of the observed LSD profiles, we averaged them in phase bins: we used bins of 0.02 for profiles with $\mathrm{He}$ or all lines, and 0.03 for profiles without $\mathrm{He}$.

We calculated a grid of $V$ profiles for each phase of observation by varying the four or five parameters mentioned above and applied a $\chi^{2}$ minimization to obtain the best fit of all phase bins simultaneously. More details of the modelling technique can be found in Alecian et al. (2008).

We applied this modelling technique to the LSD profiles obtained using all photospheric lines, He lines only, or all but $\mathrm{He}$ lines. The results obtained for each of the six models are summarized in Table 4. Figures 5 to 7 show the binned observed and modelled dipole LSD $V$ and $I$ profiles for centred dipole or offcentred dipole models, for the three groups of lines. We find that all fits reproduce the data relatively well, as indicated by the $\chi^{2}$ values (see Table 4). Nevertheless, the off-centred dipole fits are slightly better as shown by their slightly lower $\chi^{2}$ values and because the $\chi^{2}$ using $3 \sigma$ error bars on the fitted parameters $\left(\chi_{3 \sigma}^{2}\right)$ of the off-centred dipole fits are always lower than the minimum $\chi^{2}\left(\chi_{\min }^{2}\right)$ of the centred dipole fits. Therefore the small difference between the $\chi^{2}$ values of the centred and off-centred fits is significant.

We obtained values of the inclination angle $i$ that are compatible with the value $i=71 \pm 10^{\circ}$ determined by N03. When we modelled a centred dipole, we obtained values of the obliquity 
C. Neiner et al.: Detecting and modelling the magnetic field of the $\beta$ Cephei star V 2052 Ophiuchi

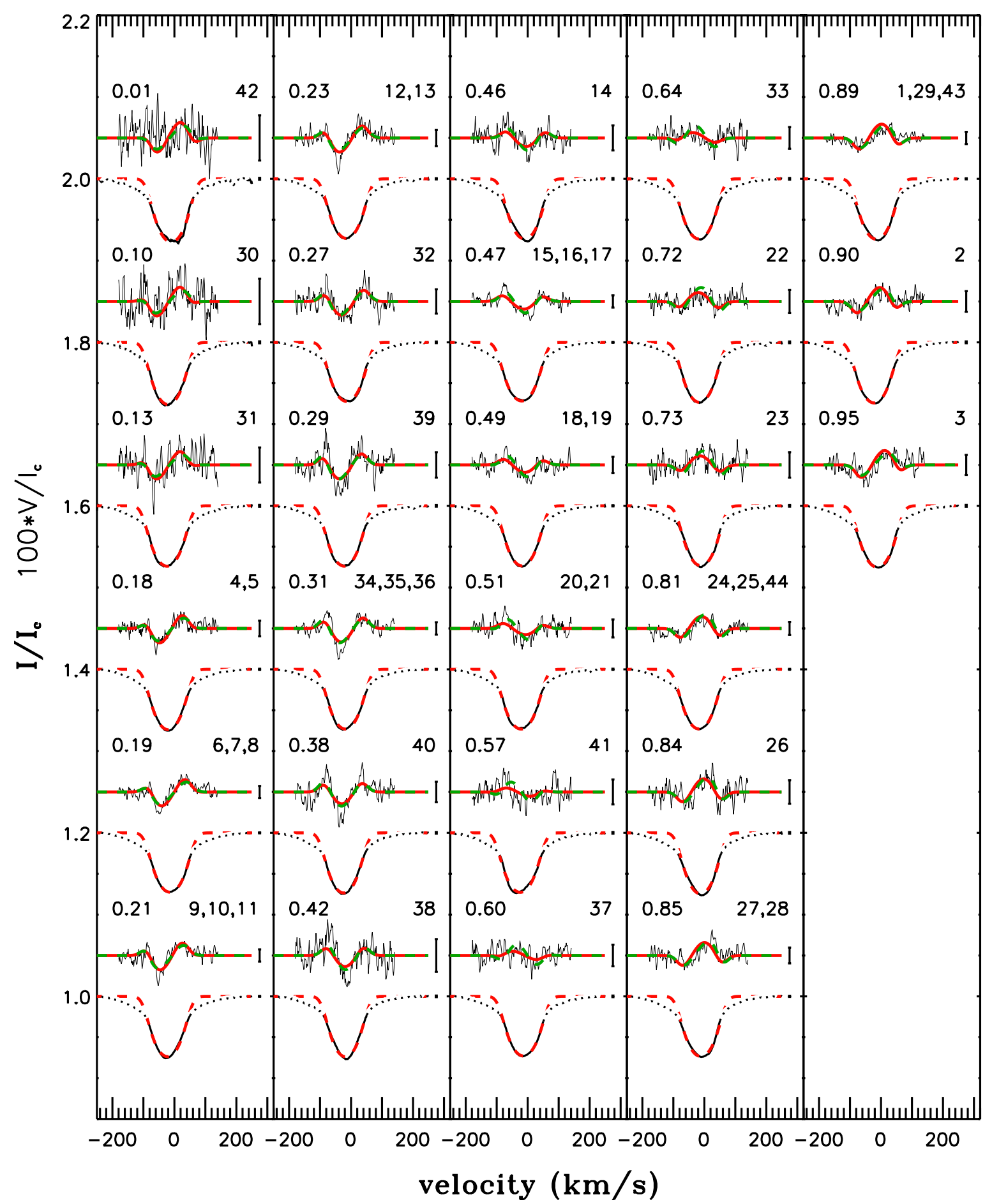

Fig. 5. Top: centred (solid red line) and off-centred (dashed green line) dipole field models superimposed on phase-binned LSD Stokes $V$ profiles (thin solid black lines) calculated for all photospheric lines. Above each profile, the rotation phase is indicated on the left and the polarimetric sets used in the phase bin is indicated on the right. Typical error bars are shown next to each profile. Bottom: corresponding modelled (dashed red line) and observed (dotted black line) phase-binned LSD Stokes I profiles.

angle $\beta$ compatible with those determined above from the (centred) dipole fit of the longitudinal field.

When we fitted an off-centred dipole, we systematically obtained an inclination angle $i$ and polar field strength $B_{\text {pol }}$ weaker and an obliquity angle $\beta$ wider than in the centred case. Again we find that the phase of the centred and off-centred dipole fit is significantly shifted compared to the UV ephemeris, but the shift is less strong in the off-centred case. Morever, we obtained a value of the decentring $d$ that is significantly different from 0 .
In the off-centred case we calculated the value of the magnetic field strength at the positive and negative magnetic poles with

$$
\left|B^{ \pm}\right|=\frac{\left|B_{\text {pol }}\right|}{(1 \mp d)^{3}} .
$$

Results are reported in Table 4. 


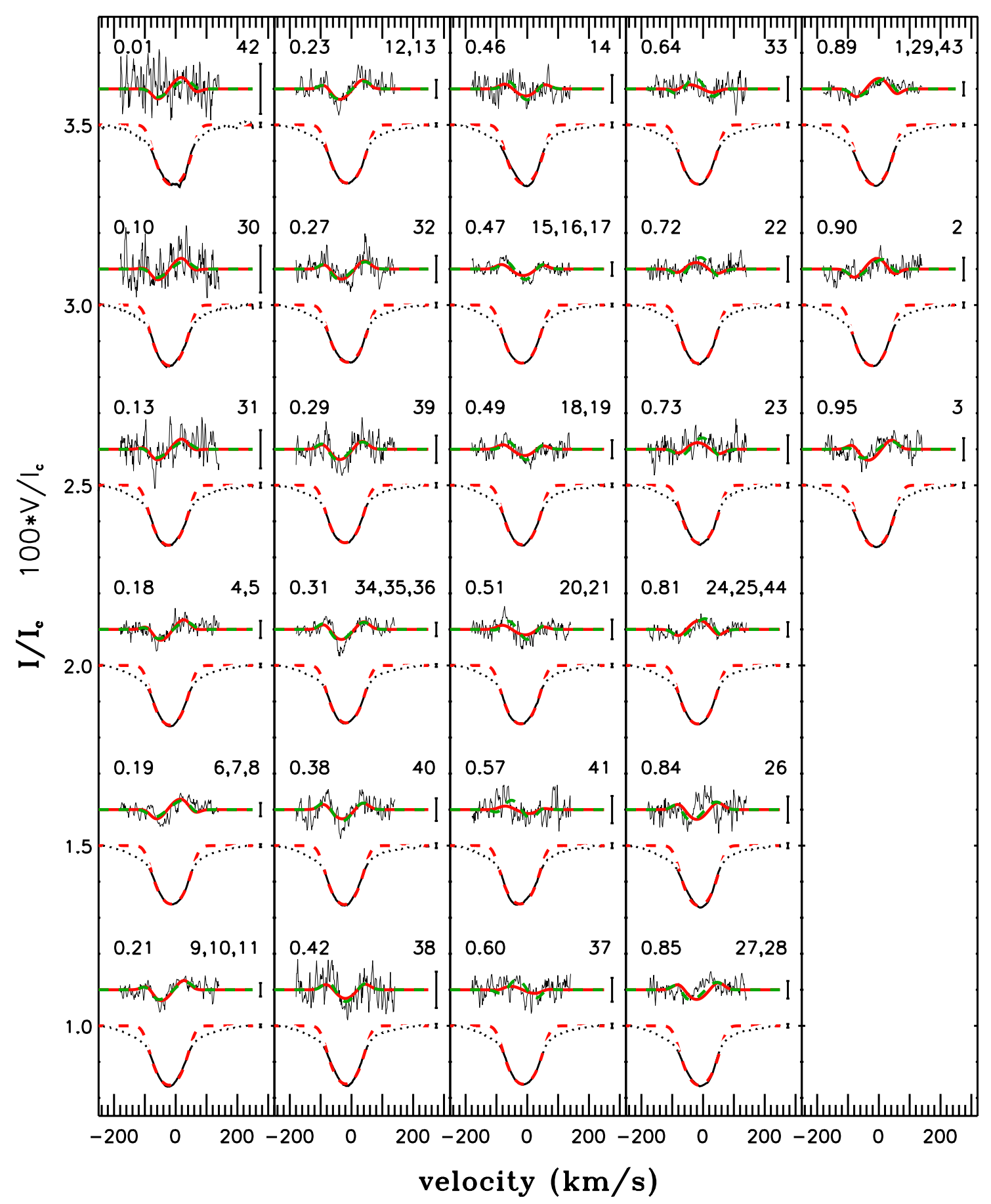

Fig. 6. Same as Fig. 5 for He lines only.

\section{Discussion}

Up to now a direct magnetic signature has been detected in only a handful of $\beta$ Cephei stars. $\beta$ Cep itself was the first $\beta$ Cephei star for which a Zeeman signature had been directly detected (Henrichs et al. 2000). Since then, signatures have been reported for $\xi^{1}$ CMa (Hubrig et al. 2006; Silvester et al. 2009; FourtuneRavard et al. 2011), 16 Peg (Henrichs et al. 2009), and $\sigma$ Lup (Henrichs et al. 2011). Other magnetic $\beta$ Cep stars have been suggested (e.g. Hubrig et al. 2009) but additional observations at higher signal-to-noise and resolution rejected those possible detections (e.g. Silvester et al. 2009; Shultz et al. 2011). In the same way, the detection of a magnetic field in the $\beta$ Cephei star $\gamma$ Peg claimed by Butkovskaya \& Plachinda (2007) has been found to be spurious by the MiMeS collaboration (Neiner et al., in prep.). With MiMeS data this star is shown to be non-magnetic at the Gauss level.

The presence of a magnetic field was known in the $\beta$ Cep star V 2052 Oph from the rotational modulation of the circular polarisation of the light of this star and from indirect evidences such as the rotational modulation of the equivalent width of the UV lines sensitive to the wind (N03), but no direct Stokes $V$ signatures had been measured so far for this star. N03 also showed that V 2052 Oph is a slightly He-rich star. Recently, Oskinova et al. (2011) detected soft X-ray emission from V 2052 Oph with $L_{\mathrm{X}} \sim 3 \times 10^{29} \mathrm{erg} \mathrm{s}^{-1}$, making it a very weakly X-ray luminous 
C. Neiner et al.: Detecting and modelling the magnetic field of the $\beta$ Cephei star V 2052 Ophiuchi

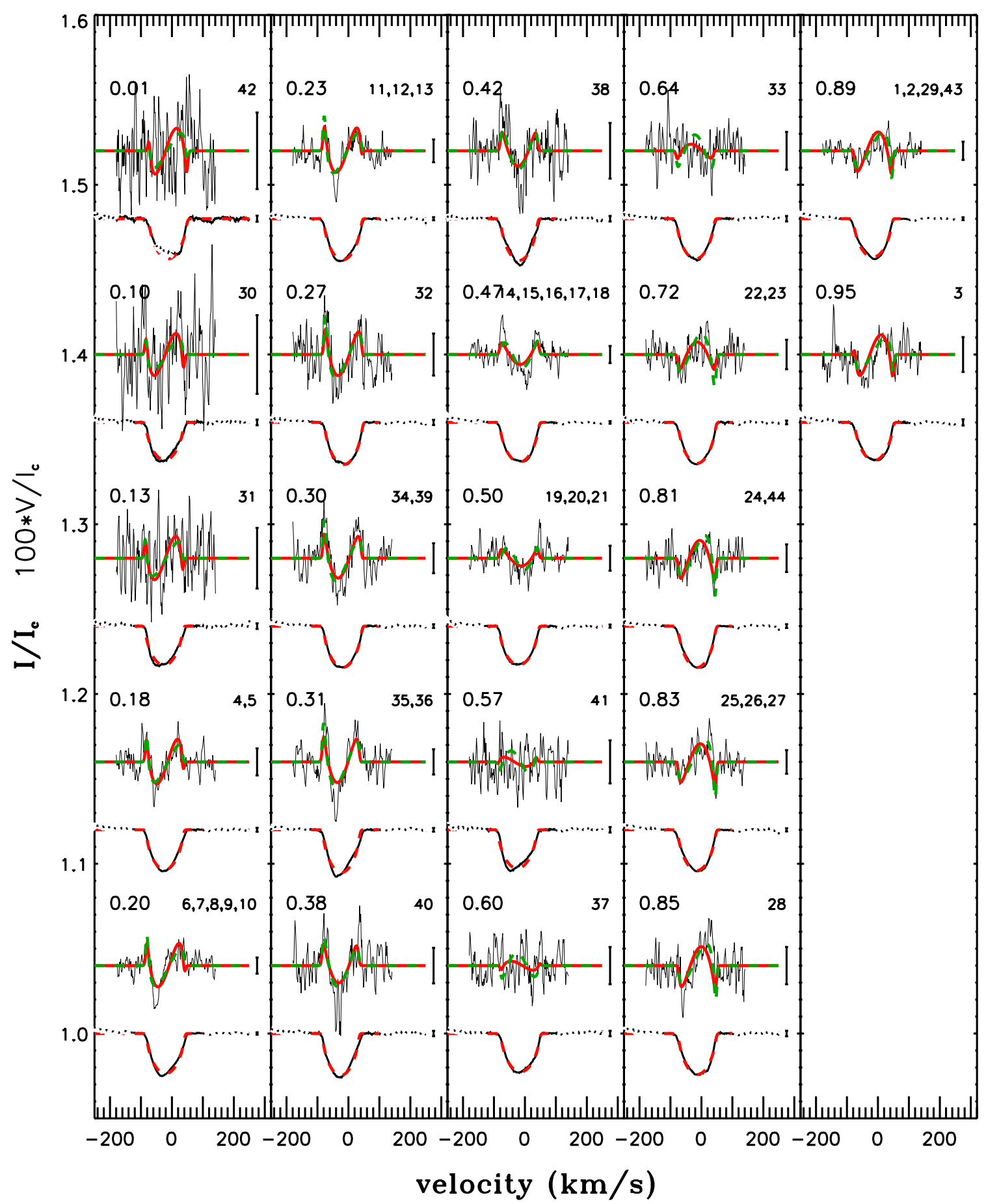

Fig. 7. Same as Fig. 5 for all photospheric lines except He.

early B-type star. Around massive stars, X-rays can be produced by shock waves in the stellar winds or by magnetically confinement of material at the magnetic equator. All this points towards the presence of a magnetic field in V 2052 Oph.

With the Narval spectropolarimeter, we were able to directly and clearly detect the Zeeman signature of the magnetic field of V 2052 Oph. This confirms that V 2052 Oph is a magnetic $\beta$ Cep star.

Moreover, the much better precision of the magnetic measurements with Narval compared to Musicos allowed us to perform a much more precise study of the magnetic field, in particular to study He lines separately.
First of all, we found that $\left|B_{0}\right|$ is different from 0 (of the order of 25-30 G). This gives constraints on the angles $i$ and $\beta$ and is consistent with the fact that two unequal minima are observed in the periodic variation of the equivalent width of UV resonance lines (see Fig. 3 of N03). The phase difference between the two maxima of this UV curve is $\Delta \phi=0.335$. For the dipole fit of the Narval longitudinal field data we found that the dipole curve crosses $B=0$ with $\Delta \phi=0.38$ when using all lines, 0.37 when using He lines only, and 0.35 when using all but He lines. Therefore, when we rejected He lines, we found a dipole fit that is more consistent with what we expect from UV data. This is confirmed because the phase of the dipole fit 
when rejecting He lines is compatible with the UV curve, while the dipole curve determined when using or adding He lines is shifted compared to the UV curve. Consequently, the dipole fit without $\mathrm{He}$ lines seems to better reproduce the observations in the UV. This is directly related to the fact that He is not uniformly distributed over the stellar surface (see below).

The geometry obtained from the observations with Narval are compatible with the results obtained indirectly from the variation of UV wind-sensitive resonance lines and with the original detection of the dipole field from rotational modulation (N03). The geometry we obtained from fitting the longitudinal field values and from fitting the Stokes $V$ profiles with a centred oblique dipole are also compatible.

Moreover, the fact that the two maxima of the UV curve of Neiner et al. (2003a) are unequal suggest that the dipole might be off-centred. Indeed, the maxima in the UV curve correspond to the magnetic equator seen edge-on (see Fig. 3 in Neiner 2007). Two unequal maxima therefore indicate that the two hemispheres of the magnetic field are different and therefore that the field is off-centred. The detection of Zeeman signatures allowed us to test this possibility. We indeed found that the $\chi^{2}$ value of the off-centred dipole fit is lower than the one of the centred dipole, with a significant value of the decentring. This confirms that V 2052 Oph indeed hosts an off-centred oblique dipole field. Off-centred dipoles have already been observed in several other types of stars, such as Ap stars or white dwarfs (e.g. Mathys 1993; Putney \& Jordan 1995) and appear in numerical simulations (Braithwaite \& Nordlund 2006).

Futhermore, theoretical work (Duez \& Mathis 2010; Braithwaite 2008) showed that the most probable magnetic mode in stellar radiation zones is a dipole but that quadrupolar equilibrium and consequently an off-centred dipole is also possible. For a specific modelling of the latter case, see Moss (1985b,a). The relative amplitude of the dipolar versus quadrupolar component has not been determined theoretically so far. V 2052 Oph could therefore serve as a prototype for constraining new theoretical work and simulations.

In addition, when comparing the centred and off-centred models for masks including He lines (Figs. 5 and 6), we found that some Stokes $V$ profiles, e.g. at phases $0.19,0.31,0.84$ and 0.85 , did not fit well, while they were relatively well-fitted when we rejected He lines (Fig. 7). Morever, both in the longitudinal field curve and in the modelled Stokes $V$ profiles, we found that the dipole seems slightly shifted when considering He lines. This can be explained by the presence of patches of helium close to the magnetic poles. These patches, close to the magnetic poles but not centred on the poles, are often observed in chemically peculiar magnetic stars (e.g. $\sigma$ Ori E). Chemical patches are a signature of the inhibition of mixing by the magnetic field and/or of the fractionation of the wind particles above the magnetic poles (Hunger \& Groote 1999).

Finally, both in the centred and off-centred dipole modelling of the Stokes $V$ profiles and in the longitudinal field analysis, we found that the $B_{\text {pol }}$ value derived when rejecting He lines is higher than when including He lines (see Tables 3 and 4). This cannot be explained by the scatter of measurements in the bottom panel of Fig. 4 only, because this scatter is already reflected in the error bars provided in Table 2 . The discrepancy could be related to a bad estimate of the sensitivity of lines in the LSD process, e.g. if the Landé factors that are available are not accurate enough, or to the fact that we neglected the broadening caused by NLTE effects in the He lines.

\section{Conclusion}

We confirm that V 2052 Oph is a magnetic $\beta$ Cephei star. It hosts a magnetic oblique dipole field with $B_{\text {pol }} \sim 400 \mathrm{G}$ and an obliquity angle $\beta \sim 35^{\circ}$ that is most likely off-centred along the magnetic axis. In addition we showed that He patches are present at the surface of the star, close to the magnetic poles.

Our study confirms that the search for magnetic candidates via indirect UV evidences (Henrichs 2001) is very useful and that the technique of the detection of a magnetic field through rotational modulation in Stokes $V$ profiles is efficient, even without clear detection of the Zeeman signature, as applied e.g. in N03 and for $\omega$ Ori in Neiner et al. (2003b).

More observations with a better time coverage and sampling would allow a better characterisation of the He patches and creating a Zeeman-Doppler map of the surface of the star. Moreover, a detailed modelling of the Stokes $I$ and $V$ profiles including pulsations, rapid rotation, and the various distribution of chemical abundances over the stellar surface would be useful to better understand the configuration of the magnetic field and starspots of V $2052 \mathrm{Oph}$.

Acknowledgements. This research has made use of the Simbad database maintained at CDS, Strasbourg, France. We wish to thank the Programme National de Physique Stellaire (PNPS) for their support. M.B. acknowledges the Fund for Scientific Research - Flanders for a grant for a long stay abroad.

\section{References}

Alecian, E., Catala, C., Wade, G. A., et al. 2008, MNRAS, 385, 391

Borra, E. F., \& Landstreet, J. D. 1980, ApJS, 42, 421

Braithwaite, J. 2008, MNRAS, 386, 1947

Braithwaite, J., \& Nordlund, А. 2006, A\&A, 450, 1077

Butkovskaya, V. V., \& Plachinda, S. I. 2007, A\&A, 469, 1069

Donati, J.-F., Semel, M., Carter, B. D., Rees, D. E., \& Collier Cameron, A. 1997, MNRAS, 291, 658

Duez, V., \& Mathis, S. 2010, A\&A, 517, A58

Fourtune-Ravard, C., Wade, G. A., Marcolino, W. L. F., et al. 2011, in Active OB stars: structure, evolution, mass loss, and critical limits, ed. C. Neiner, G. Wade, G. Meynet, \& G. Peters (Cambridge University Press), IAU Symp., 272, 180

Henrichs, H. F. 2001, in Magnetic Fields Across the Hertzsprung-Russell Diagram, ed. G. Mathys, S. K. Solanki, \& D. T. Wickramasinghe, San Francisco, USA, ASP Conf. Ser., 248, 393

Henrichs, H. F., de Jong, J. A., Donati, D.-F., et al. 2000, in Magnetic Fields of Chemically Peculiar and Related Stars, ed. Y. V. Glagolevskij, \& I. I. Romanyuk, Moscow, 57

Henrichs, H. F., Neiner, C., Schnerr, R. S., et al. 2009, in Cosmic Magnetic Fields: From Planets, to Stars and Galaxies, ed. J. E. B. K. G. Strassmeier, \& A. G. Kosovichev (Cambridge University Press), IAU Symp., 259, 393

Henrichs, H. F., Kolenberg, K., Plaggenborg, B., et al. 2011, in Active OB stars: structure, evolution, mass loss, and critical limits, ed. C. Neiner, G. Wade, G. Meynet, \& G. Peters (Cambridge University Press), IAU Symp., 272, 192 Hubrig, S., Briquet, M., Schöller, M., et al. 2006, MNRAS, 369, L61

Hubrig, S., Briquet, M., De Cat, P., et al. 2009, Astron. Nachr., 330, 317

Hunger, K., \& Groote, D. 1999, A\&A, 351, 554

Mathys, G. 1993, in Peculiar versus Normal Phenomena in A-type and Related Stars, ed. M. M. Dworetsky, F. Castelli, \& R. Faraggiana, San Francisco, USA, IAU Colloq., 138, ASP Conf. Ser., 44, 232

Morel, T., Butler, K., Aerts, C., Neiner, C., \& Briquet, M. 2006, A\&A, 457, 651

Moss, D. 1985a, MNRAS, 215, 159

Moss, D. 1985b, MNRAS, 213, 575

Neiner, C. 2007, in Active OB-Stars: Laboratories for Stellar and Circumstellar Physics, ed. A. T. Okazaki, S. P. Owocki, \& S. Stefl, San Francisco, USA, ASP Conf. Ser., 361, 91

Neiner, C., Henrichs, H. F., Floquet, M., et al. 2003a, A\&A, 411, 565 (N03)

Neiner, C., Hubert, A.-M., Frémat, Y., et al. 2003b, A\&A, 409, 275

Oskinova, L. M., Todt, H., Ignace, R., et al. 2011, MNRAS, 416, 1456

Putney, A., \& Jordan, S. 1995, ApJ, 449, 863

Shultz, M., Wade, G. A., Grunhut, J., et al. 2011, MNRAS, accepted

Silvester, J., Neiner, C., Henrichs, H. F., et al. 2009, MNRAS, 398, 1505 